\title{
Pressure-sensitive dissipation in elastomers and its implications for the detonation of plastic explosives
}

\author{
Wolfgang G. Knauss and Sairam Sundaram \\ Graduate Aeronautical Laboratories, Mail Code 105-50, California Institute of Technology, Pasadena, \\ California 91125
}

(Received 11 June 2004; accepted 21 September 2004)

\begin{abstract}
The role of binder deformation and the associated energy dissipation on the detonation sensitivity of plastically bonded explosives is considered by accounting for dilatation-sensitive viscoelastic shear response. Following the observation that pressurization can prolong the relaxation and retardation times of a viscoelastic elastomer tremendously, the implications of this phenomenon are considered for a thin layer of a model elastomer, sheared between two blocks of octahydro-1,3,5,7tetranitro-1,3,5,7-tetrazocine under deformation rates typical in detonation scenarios. The consequences of concurrent pressurization on heat generation are examined using small deformation as well as finite deformation analyses. While a dilatation-insensitive viscoelastic behavior generates notable temperature increases, they are insufficient to cause ignition of the explosive. However, taking into account the increased dissipation associated with the pressure-induced changes in the intrinsic time scale and viscosity of the elastomer leads to temperature rises on the order of $1000{ }^{\circ} \mathrm{C}$, which are consistent with "hot spots" held responsible for the initiation of detonation in the adjacent explosive grains. (C) 2004 American Institute of Physics. [DOI: 10.1063/1.1818349]
\end{abstract}

\section{INTRODUCTION AND MOTIVATION}

High explosives function by releasing large amounts of chemical energy when heated to sufficiently high temperatures. ${ }^{1}$ A common class of high explosives referred to as plastically bonded explosives (PBX) consist of grains of an energetic material (e.g., octahydro-1,3,5,7-tetranitro1,3,5,7-tetrazocine or hexahydro-1,3,5-trinitro-1,3,5-triazine held together by a thin polymer matrix. ${ }^{2}$ The decomposition of the energetic grains releases energy and gaseous products, and the expanding gaseous products accelerate the rate of reaction and strengthen the detonation front. In the case of shock wave loading, however, the high temperatures required for the initiation of chemical reaction in these explosives cannot normally be attained by a homogeneous deformation within the explosive. It is generally understood that in such cases the initiation of detonation occurs in localized, small regions within the explosive, referred to as hot spots, where the local temperatures are sufficiently high to initiate selfsustaining chemical reactions.

Several mechanisms have been proposed to explain the origin of these hot spots. These include adiabatic heating of trapped gases in cavities, local viscous heating due to void collapse, frictional rubbing between adjacent explosive grains, and fracture of and shear banding in the explosive crystals. ${ }^{3}$ Experimental evidence exists for almost all of these mechanisms, ${ }^{4}$ and in any given situation involving the detonation of an explosive by impact, one or more of these mechanisms may be dominant at various times during the initiation to detonation transition. However, one other mechanism that is particularly relevant to the class of PBX has not been studied in any significant detail. These materials are composed of explosive crystals $20-200 \mu \mathrm{m}$ in dimension, held together in an elastomeric matrix (typically a few microns thick layer around the explosive crystals). This rub- bery matrix comprises only a small fraction of the explosive (3\%-10\% by volume) but by this very fact, as will be described presently, can have a significant effect on the initiation of detonation in the explosive.

While it is generally acknowledged that localized shear deformation mechanisms are among the leading contenders for the formation of hot spots, much of the research on this front has been directed towards shear banding in the explosive crystals ${ }^{5,6}$ or frictional heating of shear crack surfaces. ${ }^{7-9}$ However, several researchers have also suggested that hot spots can be generated by mechanical deformation of the polymeric binder phase. ${ }^{10,11}$ There also appears to be some experimental evidence for this mechanism of hot spot formation: ${ }^{12-14}$ Swallowe and Field ${ }^{10,13}$ and Heavens and Field ${ }^{12}$ report photographic studies of the deformation of explosive granules as well as polymers between impacting glass anvils and conclude that certain polymers exhibit a sensitizing action on explosives. In drop-weight tests these polymers show a rapid reduction in the load required to produce catastrophic failure of the sample. Postimpact microscopic examinations of the polymers reveal that the catastrophic failure of the samples is associated with shear in localized bands and led the authors to conclude that this failure mode is responsible for the polymers' sensitizing action. In a later section we discuss the connection between this observation and the results of our computations.

Elastomeric binders used in high explosives typically display rubbery behavior at atmospheric conditions and hence possess characteristically low shear moduli (relative to the bulk modulus). For example, a thermoplastic polyurethane (commercially sold as Estane) used as a binder in many high explosives, has a rubbery modulus of $\approx 2 \mathrm{MPa}$ at $100 \%$ elongation and $4 \mathrm{MPa}$ at 300\% elongation (B. F. Goodrich data). Under shock wave loading, the thin binder layer 


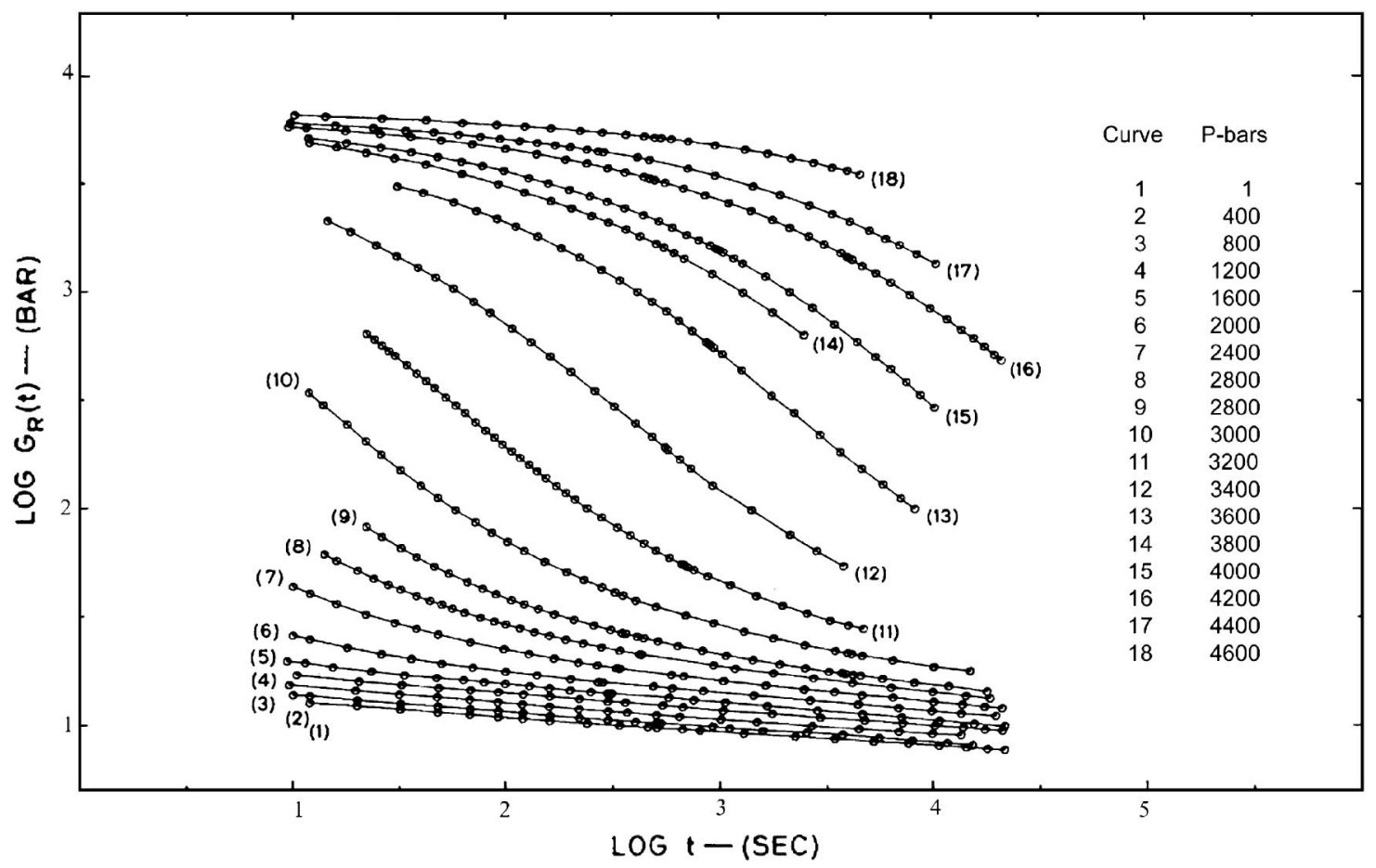

FIG. 1. Effect of pressure on the relaxation modulus in shear of the elastomer Hypalon 40 [1 bar=curve (1); 4.6 kbar=curve (18)]. (Reproduced with permission of the publisher Elsevier from Fillers and Tschoegl, Ref. 24).

between grains is subjected to high pressure and shear loads. Because binder is present as very thin layers sandwiched between harder explosive crystals, it can also experience locally large shear strain rates.

Current mechanical models of explosives which attempt to describe the heterogeneous microstructure of plastic explosives typically in terms of the rubbery behavior of the binder. ${ }^{15}$ The associated low modulus values permit the binder-and hence the explosive- to attain only small shear stress levels, which are associated with correspondingly low inelastic dissipation values. On the other hand, it is known that elastomers undergo tremendous stiffening under imposed pressure as a result of a change in the intrinsic time scale (see Fig. 1).

The influence of volumetric change on the rate or time dependent response of polymers is well recognized, though usually in connection with its effect on the value of the glass transition temperature. ${ }^{16-19}$ Connected, at least qualitatively, with the notion of variability in free volume which changes the degrees of freedom for motion of chain segments, small changes in volume can contribute to relatively large changes in the creep or relaxation response of polymers. This effect has been explored for structural polymers below the glass transition by Knauss and Emri ${ }^{20}$ and Losi and Knauss. ${ }^{21}$ The nearly universal phenomenon of time-temperature trade-off for linearly viscoelastic behavior has been characterized very successfully by Tobolsky and Eyring ${ }^{22}$ and by Williams, Landel, and Ferry ${ }^{23}$ through a free volume formulation for the time-temperature shift factor [defined in the well known Williams, Landel, and Ferry (WLF) equation].

We refer to this pressure induced effect as "stiffening," because identical mechanical deformation within the material responds with a (much) higher modulus. This result is the consequence of eliciting molecular response at a reduced internal or intrinsic time scale in that the pressure transposes the material towards or into the glassy state. If this transposition is such that at the external (experimental) time scale the material responds with the relaxation times mostly in the middle of the rubber-to-glass transition, then it will, commensurately, produce maximal dissipation in any deformation or relaxation process. Thus, what we refer to as pressure-induced stiffening is automatically associated with pressure-induced increases in the viscosity.

With respect to rubbery materials, Tschoegl and co-workers ${ }^{24,25}$ conducted extensive studies on the influence of volume changes via mechanical pressure on the shear relaxation moduli of several elastomers and have reported modulus increases of up to three orders of magnitude under pressure increases as low as $0.5 \mathrm{GPa}$. For example, Hypalon 40 (a lightly filled chlorosulfonated polyethylene) exhibits a shear modulus of $\approx 1 \mathrm{MPa}$ at $25{ }^{\circ} \mathrm{C}$ under atmospheric pressure. Under a pressure of $0.5 \mathrm{GPa}$ the shear modulus increases to $630 \mathrm{MPa}^{26}$ By comparison, shock loading typically raises pressure to levels on the order of a few gigapascals before the initiation of detonation. Under such conditions, the stiffening or hardening of the rubbery binder would lead to much higher shear stress levels and correspondingly higher levels of inelastic dissipation in the binder than response under atmospheric pressure would indicate. This elevated and pressure-augmented dissipation and consequent heating of the binder could produce local hot spots to ignite the adjacent explosive crystals. Furthermore, the increased levels of shear stress sustained by the binder also cause increased shear stresses in the explosive crystals and are likely to cause fracture of the crystals and thus form or support other sources of hot spots. On the other hand, in- 
creasing times and temperatures evoke the softer characteristics of the binder (response to the longer relaxation times) and thus tend to lower the stress and dissipation levels. It is thus the objective of this study to examine these competing effects and their implications for the initiation of detonations in plastic-bonded explosives. It may be noted here that the data of Tschoegl and co-workers ${ }^{24,25}$ are from static experiments whereas the deformation of the binder in plastic explosives takes place dynamically. The dynamic response of polymers under impact loading conditions is a subject of past and current study (see, for example, Gupta ${ }^{27}$ ); however, such experiments still do not provide a comprehensive description of polymer response over the large pressure-temperature space that would really be needed for the kind of analysis described in this paper. We, therefore, base our study on the generally accepted understanding in polymer mechanics that the relaxation response of the polymer over a large time scale from nanoseconds to hours derived from the timetemperature-pressure superposition behavior under static loading conditions is valid under dynamic conditions as well. Over the past half century there has been no investigation nor data that have contradicted this concept. Should future examination of this question materialize, we expect that deviations from the current point of view are quantitatively limited but without a total breakdown of the concept.

The paper is structured as follows. We begin with a brief review of the experimental results of Tschoegl and co-workers $^{24,25}$ (henceforth referred to as Tschoegl, Fillers, and Moonan or TFM) demonstrating the pressure-induced increase in the shear relaxation moduli (stiffening) of elastomers. These experimental characterizations are then used in an analysis of the shearing of a thin, elastomeric binder layer sandwiched between hard, explosive grains while under pressure. The problem is first analyzed in a small deformation framework which allows a clearer understanding of the primary effects, and then in a finite deformation framework. The purpose of this dual approach is to determine that the fundamental system response is not so much the result of detailed mechanics modeling but that suitable pressure sensitivity in the viscoelastic binder provides the dominant effect. For both analyses, results of the calculations are presented with discussions of the relevance to the detonation of plastic explosives.

\section{EXPERIMENTAL RESULTS ON PRESSURE SENSITIVE RELAXATION}

To the best of our knowledge Fillers and Tschoegl ${ }^{24}$ and Moonan and Tschoegl ${ }^{25}$ (TFM) have obtained the only comprehensive measurements of the shear relaxation modulus of elastomeric materials as a function of both pressure and temperature, though others ${ }^{28-30}$ have explored related effects in polymers below their glass transition temperatures (rigid polymers). Through an extensive set of measurements TFM demonstrated that polymeric materials stiffen considerably with increasing pressure by "translating them into a shorter time domain" and that the time-dependent modulus curves display a time-pressure superposition behavior similar to, and, essentially, in addition to, the well known timetemperature WLF superposition behavior. Specifically, seg- ments of the time-dependent relaxation moduli curves of a polymer at different pressure levels are shifted relative to each other along a logarithmic time axis and hence can be represented by a single master curve and a pressure dependent shift factor $a_{P}(P)$ which represents the shifting of the master curve with respect to the pressure $P$.

The combined effects of pressure and temperature are then represented through a master curve at a reference temperature and reference pressure $\left(T_{0}, P_{0}\right)$ combined with a pressure and temperature dependent shift function $a_{T, P}(T, P)$. Such a material is referred to as a thermorheologically- and piezorheologically simple material. Figure 1 shows a set of (isothermal) shear relaxation modulus measurements at $25^{\circ} \mathrm{C}$ and different pressures for Hypalon 40. Figure 2 shows the corresponding master curve reduced to $T_{0}$ $=25{ }^{\circ} \mathrm{C}$ and $P_{0}=1$ bar resulting from the shifting of isothermal and isobaric curves in Fig. 1. The inset shows the corresponding shift function $a_{T, P}$.

For those not routinely versed in thermoviscoelastic behavior of polymers a comment is in order that addresses the relation between the material characterization effected quasistatically in the Fillers/Tschoegl work and the dynamical conditions associated with the high rate pressurization problem typical for explosives. One first notes that, in principle, constitutive behavior is formulated independently of the mode or speed of deformation: Inertial considerations do not enter into the description of constitutive relations. Like any elastic material characterization, viscoelastic material descriptions apply equally to static and dynamic problems. The difference between the two sets of materials is that the latter are implicitly deformation rate sensitive regardless of whether material inertia plays a role or not, and the same is not true for the former.

On the other hand, an approximation commonly used in engineering problems where volumetric deformations are encountered, will be employed here. This approximation treats the bulk response as elastic so that no delayed volume changes occur under rapid pressure applications. This is a mild substitution for real material behavior because the viscoelastic effect on volume deformation is orders of magnitude smaller than the shear behavior. ${ }^{31-33}$

We write the linearly viscoelastic constitutive description as

$$
\sigma_{i j}=2 \int_{-\infty}^{t} \mu_{0}(t-\xi) \frac{\partial \epsilon_{i j}}{\partial \xi} d \xi+\delta_{i j} \int_{-\infty}^{t} K_{0}(t-\xi) \frac{\partial \epsilon_{k k}}{\partial \xi} d \xi
$$

where $\mu_{0}(t)$ and $K_{0}(t)$ are the time-dependent shear and bulk relaxation moduli at some reference temperature and pressure $\left(T_{0}, P_{0}\right)$.

If pressure and temperature change during the course of the deformation, the material relaxation times are affected incrementally in time to produce a stiffening or accelerated softening of the binder in addition to the normally intrinsic time-dependent relaxation. The effect of changing temperature and pressure is included through the concept of an intrinsic material time defined as 


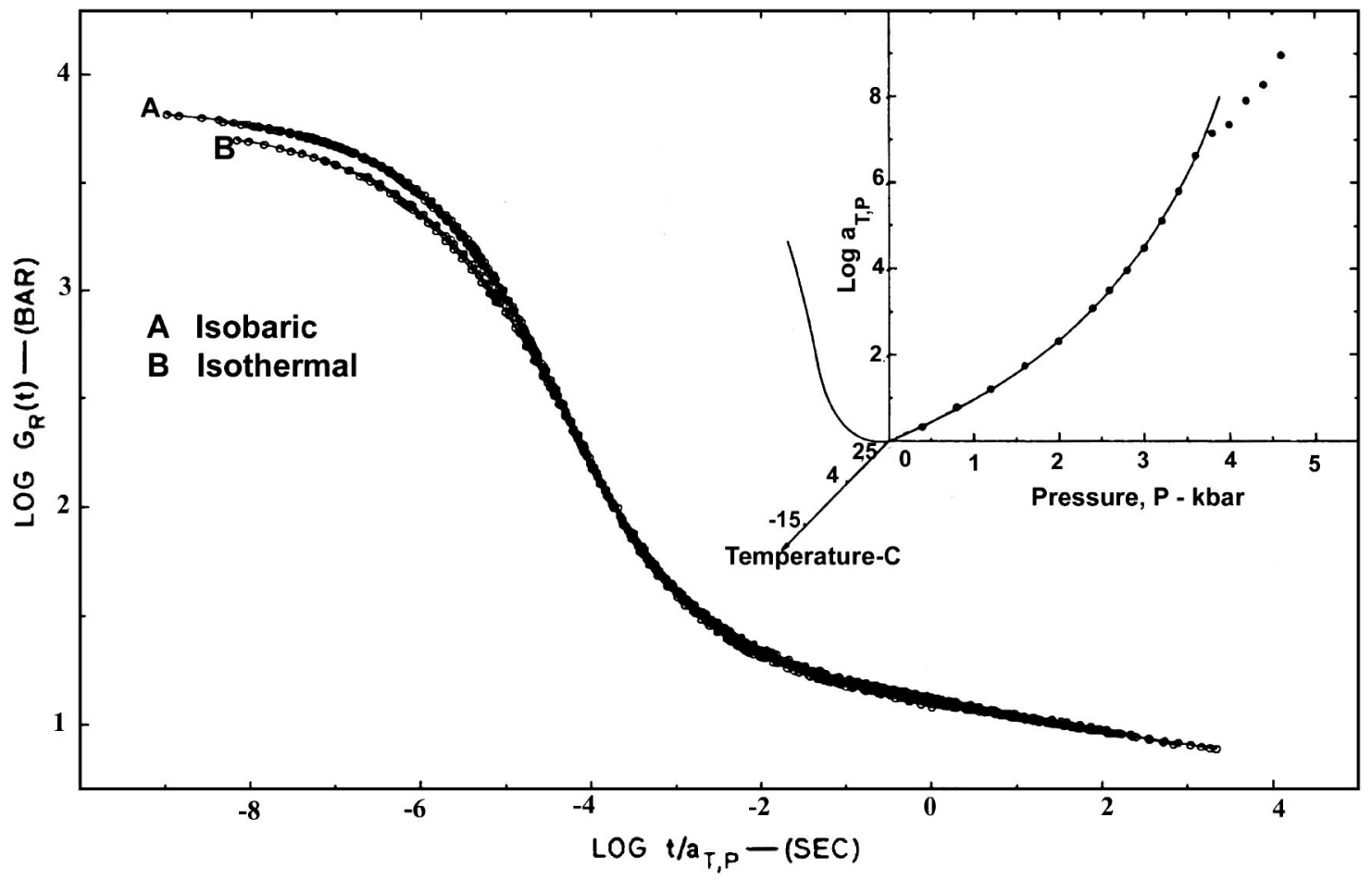

FIG. 2. Master relaxation curve and shift function for Hypalon 40 (from Fillers and Tschoegl, Ref. 24). This is a single curve representation of the pressure effects shown in Fig. 1 and also includes the effect of temperture. The inset figure shows the shift function $a_{T, P}$ that, together with the master curve, summarizes the effect of temperature and pressure on the shear relaxation modulus of the elastomer.

$$
t^{\prime}=\int_{0}^{t} \frac{d t}{a_{T, P}}
$$

where

$$
a_{T, P}=a_{T, P}(T, P)=a_{T, P}[T(t), P(t)]
$$

is the temperature and pressure dependent shift function as defined with respect to the reference conditions on temperature and pressure $\left(T_{0}, P_{0}\right)$ (e.g., see inset in Fig. 2). The (nonlinear) viscoelastic formulation is then written as

$$
\sigma_{i j}=2 \int_{-\infty}^{t} \mu_{0}\left(t^{\prime}-\xi^{\prime}\right) \frac{\partial \epsilon_{i j}}{\partial \xi} d \xi+\delta_{i j} \int_{-\infty}^{t} K_{0}\left(t^{\prime}-\xi^{\prime}\right) \frac{\partial \epsilon_{k k}}{\partial \xi} d \xi
$$

The expression for the shift function $a_{T, P}$ given by TFM is

$$
\ln a_{T, P}=-\frac{c_{1}\left\{[T-\theta(P)]-T_{0}\right\}}{c_{2}+[T-\theta(P)]-T_{0}},
$$

where

$$
\theta(P)=c_{3} \ln \left[\frac{1+c_{4} P}{1+c_{4} P_{0}}\right]-c_{5} \ln \left[\frac{1+c_{6} P}{1+c_{6} P_{0}}\right]
$$

The reader is referred to Refs. 24 and 25 for details on the derivation of Eqs. (5) and (6) as well as on obtaining the values of the parameters $c_{i}$.

\section{A. Modification of the TFM shift function for higher pressures}

The TFM expression for the temperature and pressure dependent shift function $a_{T, P}$ [Eq. (5)] is a modification of the WLF (Ref. 23) expression for the shift function which can be based on free volume changes in connection with temperature changes. The TFM expression includes the effect of pressure on free volume through the compressibility, and is incorporated into the WLF expression as an apparent pressure-induced temperature drop denoted by $\theta(P)$. Increasing the pressure is, thus, equivalent to decreasing the temperature. However, a feature of the free volume based WLF theory is that for temperatures below the glass transition temperature $T_{g}$ the free volume remains (nearly) frozen at its value corresponding to $T_{g}$. Correspondingly, the value of the shift function does not change much below $T_{g}$. The implication for the TFM model would be that, for $[T-\theta(P)] \leqslant T_{g}$, the value of $\ln a_{T, P}$ remains saturated at or near the value

$$
\ln a_{T_{g}, P}=-\frac{c_{1}\left\{\left[T_{g}-\theta(P)\right]-T_{0}\right\}}{c_{2}+\left[T_{g}-\theta(P)\right]-T_{0}} .
$$

The modified TFM shift function is schematically illustrated in Fig. 3.

For the material whose master relaxation modulus curve is shown in Fig. 2, the glass transition temperature (at atmospheric pressure $P_{0}$ ) is $\approx-20{ }^{\circ} \mathrm{C}$ so that, at the reference temperature $T_{0}\left(=25^{\circ} \mathrm{C}\right)$, the shift function might be expected to saturate at a pressure corresponding to $\theta(P)=T_{0}$ $-T_{g}=45^{\circ} \mathrm{C}$. The value of the pressure corresponding to this value of $\theta(P)$ is $\approx 0.4 \mathrm{GPa}$ and it can be seen from Fig. 2 that the shift function indeed begins to deviate from the TFM 


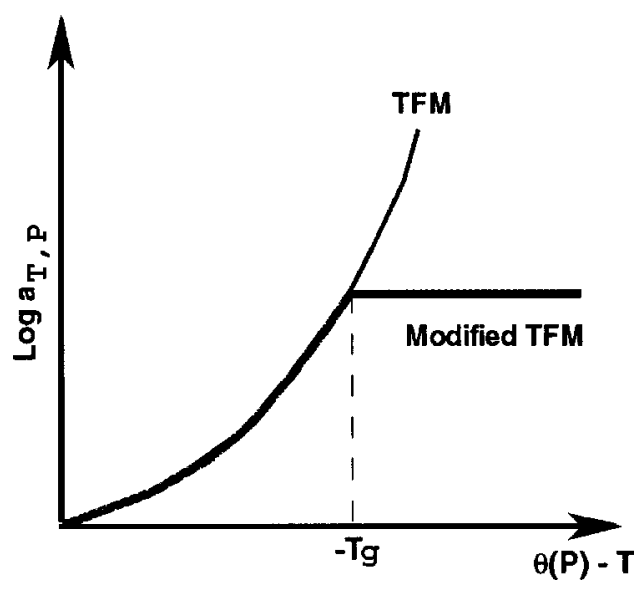

FIG. 3. Proposed modification to the TFM shift function [Eq. (5)] to account for saturation of pressure-stiffening effects at higher pressures (as well as tempertures below $T_{g}$ ).

expression near this pressure. This modification to the TFM expression cannot be overlooked for the present purposes because the stress states of primary concern typically involve pressures higher than $0.4 \mathrm{GPa}$ and use of the referenced, nonmodified TFM expression would be likely to cause unrealistic pressure-induced shifting of the relaxation behavior.

In the following section, we consider the problem of a thin layer of elastomeric binder being sheared under imposed pressure between two relatively hard (semi-infinite) grains of explosive. We examine this first in the context of small deformations with the constitutive characterization given by Eq. (4).

\section{SMALL DEFORMATION ANALYSIS OF BINDER DEFORMATION}

When a (granular) composite such as PBX deforms, highly inhomogeneous deformations occur at the scale of the explosive grains. Where grain-to-grain contact is not the dominant form of force or stress transmission. Deformation gradients are highest between grains, especially under local shear deformations, since these involve the lowest stiffness characteristics of the composite. Consider then a thin layer of viscoelastic binder sandwiched between two relatively large grains of explosive and undergoing shear deformation (Fig. 4). Our objective is to evaluate the dynamic stressdeformation behavior of this analog binder material. This includes the evaluation of the temperature increase in the binder as a result of viscoelastic and pressure-augmented dissipation as well as the resulting temperatures in the adjacent explosive grains. The binder generally forms a thin layer between explosive grains and is subject to stress-waves traveling through the explosive composite. The transit time of these waves (pressure jump) through the binder layer is typically on the order of a few nanoseconds whereas the time scales leading to detonation are on the order of tens of microseconds. Hence it is appropriate to study the homogeneous shear deformation behavior of the binder. In the following development, the nonlinear constitutive relation for the polymeric binder expressed as an integral formulation in Eq. (4) will be rederived in rate form to facilitate the numeri-

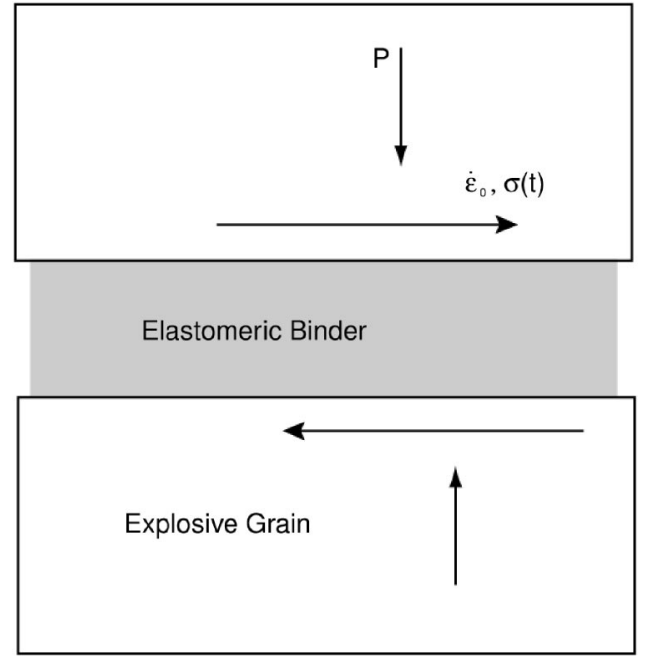

FIG. 4. Configuration for small deformation analysis of binder shearing. A thin binder layer is sandwiched and sheared between two layers of explosive grains under pressure $\mathrm{P}$ and shear strain rate $\dot{\epsilon}_{0}$.

cal evaluation of the inelastic dissipation rate which determines the temperature increase in the binder during deformation.

\section{A. Rate equations and constitutive modeling}

Let the time-dependent behavior of the viscoelastic binder at the reference temperature and pressure $\left(T_{0}, P_{0}\right)$ be characterized by a shear relaxation modulus function $G(t)$ in the form of a Prony series

$$
G(t)=\sum_{i=1}^{N} G_{i} \exp \left(-t / \tau_{i}^{0}\right) .
$$

Here $\tau_{i}^{0}$ are specific relaxation times at the reference temperature and pressure $\left(T_{0}, P_{0}\right)$ and $G_{i}$ are the corresponding moduli. The material may have, in fact, a very large number of individual relaxation mechanisms and corresponding relaxation times, but for realistic computational purposes only a limited number are necessary to adequately model the overall relaxation behavior over the entire timescale of the relaxation process. Furthermore, for a thermorheologically and piezo-rheologically simple material the relaxation times at some other temperature and pressure $(T, P)$ are given by

$$
\tau_{i}(T, P)=\tau_{i}^{0} a_{T, P},
$$

where $a_{T, P}=a_{T, P}(T, P)$ is the shift function of Eq. (3) discussed in the preceding section. The representation of Eq. (9) corresponds to a shifting of the $\ln [G(t)]-\ln [t]$ curve (Fig. 2) by $\ln \left(a_{T P}\right)$ along the $\ln [t]$ axis. We again note here that, in general, higher temperatures tend to accelerate relaxation whereas increased pressures have the opposite effect.

For representation purposes, consider a MaxwellWiechert model as shown in Fig. 5 consisting of $N$ parallel Maxwell elements. Let $G_{i}$ represent the individual spring moduli and $\eta_{i}^{0}$ the individual dashpot viscosities. If one defines 


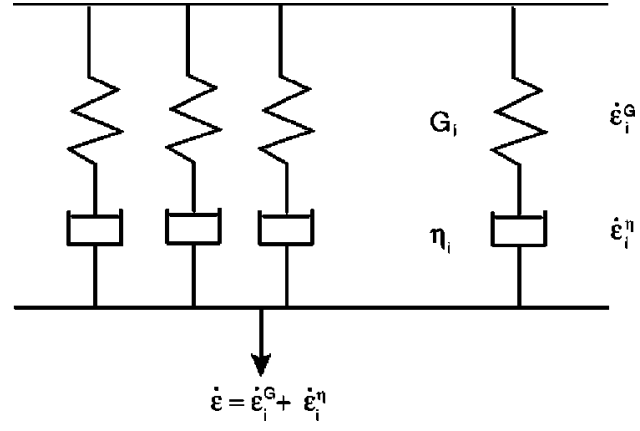

FIG. 5. Maxwell-Wiechert model representation of the mechanical behavior of the binder corresponding to the Prony series representation of Eq. (8).

$$
\tau_{i}^{0}=\eta_{i}^{0} / G_{i}
$$

then the overall relaxation modulus in shear of the model is given by Eq. (8) and hence we use this Maxwell-Wiechert model as the mechanical spring-dashpot analog for the binder to calculate the rate of viscoelastic energy dissipation during the binder deformation.

Since the individual spring-dashpot elements are in parallel, each element undergoes identical deformation. The individual dashpot viscosities, however, change during the deformation according to

$$
\eta_{i}(t)=\eta_{i}^{0} a_{T, P}(t)
$$

which is equivalent to Eq. (9).

In the following analysis, shear stresses are denoted by $\sigma$ instead of $\tau$ to avoid confusion with the relaxation times $\tau_{i}$. Correspondingly, shear strains are represented by $\epsilon$. Let the overall rate of straining of the assembly at any instant be $\dot{\boldsymbol{\epsilon}}(t)$. Then, for each spring-dashpot element $i$ we write

$$
\dot{\boldsymbol{\epsilon}}(t)=\dot{\epsilon}_{i}^{G}(t)+\dot{\epsilon}_{i}^{\eta}(t),
$$

where $\dot{\epsilon}_{i}^{G}$ and $\dot{\epsilon}_{i}^{\eta}$ represent the rate of straining of the spring and dashpot element, respectively.

If $\sigma_{i}$ represents the stress in this element, then

$$
\dot{\epsilon}_{i}^{G}(t)=\frac{\dot{\sigma}_{i}(t)}{G_{i}}
$$

and

$$
\dot{\epsilon}_{i}^{\eta}(t)=\frac{\sigma_{i}(t)}{\eta_{i}^{0} a_{T, P}(t)}=\frac{\sigma_{i}(t)}{G_{i} \tau_{i}^{0} a_{T, P}(t)} .
$$

Hence, combining Eqs. (12), (13), and (14) results in

$$
\dot{\boldsymbol{\epsilon}}(t)=\frac{\dot{\sigma}_{i}(t)}{G_{i}}+\frac{\sigma_{i}(t)}{G_{i} \tau_{i}^{0} a_{T, P}(t)},
$$

which may be rewritten as

$$
\dot{\sigma}_{i}(t)=G_{i} \dot{\boldsymbol{\epsilon}}(t)-\frac{\sigma_{i}(t)}{\tau_{i}^{0} a_{T, P}(t)} .
$$

Equation (16) can be integrated numerically to give $\sigma_{i}(t)$. The rate of viscoelastic dissipation in the $i$ th element is then
TABLE I. Values of Prony series parameters $\tau_{i}^{0}$ and $G_{i}$ used to fit the experimental shear relaxation modulus data for Hypalon 40 (see Ref. 24 and Fig. 2).

\begin{tabular}{cc}
\hline \hline $\begin{array}{c}\tau_{i}^{0} \\
(\mathrm{~s})\end{array}$ & $\begin{array}{c}G_{i} \\
(\mathrm{~Pa})\end{array}$ \\
\hline $0.100 \times 10^{-8}$ & $0.110 \times 10^{9}$ \\
$0.838 \times 10^{-8}$ & $0.803 \times 10^{8}$ \\
$0.702 \times 10^{-7}$ & $0.712 \times 10^{8}$ \\
$0.588 \times 10^{-6}$ & $0.213 \times 10^{9}$ \\
$0.492 \times 10^{-5}$ & $0.204 \times 10^{9}$ \\
$0.412 \times 10^{-4}$ & $0.658 \times 10^{8}$ \\
$0.346 \times 10^{-3}$ & $0.833 \times 10^{7}$ \\
$0.289 \times 10^{-2}$ & $0.217 \times 10^{7}$ \\
$0.242 \times 10^{-1}$ & $0.395 \times 10^{6}$ \\
$0.203 \times 10^{+0}$ & $0.400 \times 10^{6}$ \\
$0.170 \times 10^{+1}$ & $0.100 \times 10^{6}$ \\
$0.143 \times 10^{+2}$ & $0.400 \times 10^{6}$ \\
$0.119 \times 10^{+5}$ & $0.900 \times 10^{6}$ \\
\hline \hline
\end{tabular}

$$
\dot{W}_{i}^{v}(t)=\sigma_{i}(t) \dot{\epsilon}_{i}^{\eta}(t)=\frac{\left[\sigma_{i}(t)\right]^{2}}{G_{i} \tau_{i}^{0} a_{T, P}(t)}
$$

and the total stress and dissipation rate in the material are found, respectively, as the sums

$$
\sigma(t)=\sum_{i=0}^{N} \sigma_{i}(t)
$$

and

$$
\dot{W}^{v}(t)=\sum_{i=0}^{N} \dot{W}_{i}^{v}(t)=\sum_{i=0}^{N} \frac{\left[\sigma_{i}(t)\right]^{2}}{G_{i} \tau_{i}^{0} a_{T, P}(t)} .
$$

If we assume, for the moment, that there is no conduction of heat to the adjacent explosive grains, the rate of temperature rise of the homogeneously deforming binder is given by

$$
\dot{T}(t)=\beta \frac{\dot{W}^{v}(t)}{(\rho C)_{b}},
$$

where $\rho$ and $C$ are the density and specific heat capacity of the binder, respectively, both of which are taken to be constant with respect to time, temperature, and pressure for purposes of this calculation and $\beta$ is the fraction of the inelastic dissipation contributing to heating of the binder. For purposes of our analysis we assume that all inelastic dissipation is converted to heating of the binder $(\beta=1)$. The temperature history of the binder is then represented by

$$
T(t)=T_{0}+\int_{0}^{t} \frac{\dot{W}^{v}(\xi)}{\rho C} d \xi .
$$

A Prony series representation of the form of Eq. (8) is evaluated for the master relaxation curve $G(t)$ in Fig. 2 by choosing 13 relaxation times spaced approximately one decade apart. The resulting thirteen individual $G_{i} \mathrm{~s}$ and $\tau_{i}^{0} \mathrm{~s}$ are listed in Table I. The density $\rho$ for the binder, under atmospheric conditions, is $1150 \mathrm{~kg} / \mathrm{m}^{3}$ and the specific heat ca- 
pacity $C$ is $300 \mathrm{~J} /(\mathrm{kg} \mathrm{K})$ and taken to be independent of temperature and pressure for reasons of simplicity.

\section{B. Temperature and energy considerations}

As the binder undergoes deformation, the viscous dissipation causes the temperature of the binder to increase. If one assumes that no heat is lost to the adjacent explosive grains, the temperature increase of the binder may be evaluated from the evolution equation

$$
\dot{T}_{b}=\frac{\dot{W}^{v}}{(\rho C)_{b}},
$$

where $\dot{W}^{v}$ is the total dissipation rate and $(\rho C)_{b}$ is the heat capacity per unit volume of the binder. However, to determine the feasibility of forming hot spots in the region around the deforming binder, it is necessary to consider the heat conduction into adjacent explosive grains. Thus, to properly estimate the temperature distribution in the explosive grains adjacent to the binder, it is, in principle, necessary to also solve the associated heat conduction equation in the grains subject to appropriate boundary conditions at the binderexplosive interface. Such an approach would also necessitate a spatial solution of the temperature field within the binder thickness. However, since we have resorted to a consideration of homogeneous deformation of the binder as a way of reducing the complexity involved in a full solution without sacrificing much by way of physical insight and quantitative understanding, we present, in the same spirit, a simplified but sufficiently accurate treatment of the conduction issue.

It can be estimated from basic heat diffusion theory that at any time $t$ about $90 \%$ of the heat content in the explosive grain will be contained within a distance of $\sqrt{4 \alpha_{e} t}$ from the interface with the binder, where $\alpha_{e}$ is the thermal diffusivity of the explosive grain. For the time span of $10 \mu \mathrm{s}$ for which computational results are evaluated later on, this distance is $\approx 3 \mu \mathrm{m}$. Since the binder layer thickness $h_{b}$ is on the order of $20 \mu \mathrm{m}$, it is reasonable to assume that no significant amount of conduction will have occurred far into the explosive grains to warrant a full heat diffusion analysis. The temperature of the explosive grain just adjacent to the binder will thus be very close to the binder temperature evaluated by using the adiabatic rate equation Eq. (22). However, we account for heat conduction approximately by considering a uniformly heated zone of length $h_{e}(t)=0.5 \sqrt{4 \alpha_{e} t}$ into the explosive grains (see Fig. 6). Thus, the temperature history of the binder and explosive just adjacent to the binder may be calculated from

$$
\dot{T}=\frac{h_{b} \dot{W}^{v}}{h_{b}(\rho C)_{b}+2 h_{e}(t)(\rho C)_{e}} .
$$

We next account for the chemical energetics of the explosive grains. If $f$ represents the mass fraction of reaction products then the energy release per unit volume due to chemical reaction is given by

$$
\dot{W}^{e}=\dot{f} \rho_{e} \Delta H,
$$

where $\Delta H$ represents the heat of detonation, and

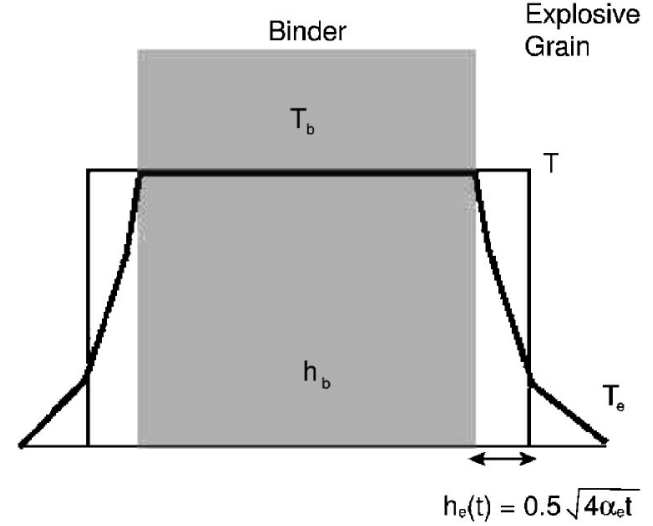

FIG. 6. Approximate thermal analysis for heat conduction from the deforming binder layer into the adjacent explosive grains. The dimension $h_{e}(t)$ represents the approximate thickness of the layer in the explosive grains where most of the conducted heat resides at any time $t$.

$$
\dot{f}=(1-f) \nu \exp -\frac{\Delta G}{k T}
$$

represents the progress of the reaction in terms of single-step Arrhenius kinetics. In the latter expression, $\Delta G$ signifies an activation energy for the reaction and $\nu$ is a pre-exponential factor generally identified with a characteristic vibrational frequency for the atoms in the explosive grains. Since our objective is not a detailed calculation of detonation reaction zones, but rather a study of characteristic features of the response of the energetic solid, we consider this one-step energetics representation sufficient. The temperature history of the binder and the explosive grains just adjacent to the binder is now determined by

$$
\dot{T}=\frac{h_{b} \dot{W}^{v}+2 h_{e}(t) \dot{W}^{e}}{h_{b}(\rho C)_{b}+2 h_{e}(t)(\rho C)_{e}} .
$$

\section{Computational results and discussions}

The evolution equations of the previous section are converted to a finite-difference form for numerical implementation. A time step of $1 \mathrm{~ns}$ is used throughout the analysis and a total deformation time of $10 \mu \mathrm{s}$ is considered since, typically, detonations in explosives initiate at time scales on the order of microseconds. The relevant parameter values are taken as follows: ${ }^{34,8} h_{b}=20 \mu \mathrm{m}, \Delta H=5.5 \times 10^{6} \mathrm{~J} / \mathrm{kg}, \Delta G$ $=2.0 \mathrm{eV}, \nu=10^{14} \mathrm{~s}^{-1}, \rho_{b}=1150 \mathrm{~kg} / \mathrm{m}^{3}, \rho_{e}=1900 \mathrm{~kg} / \mathrm{m}^{3}, C_{b}$ $=300 \mathrm{~J} /(\mathrm{kg} \mathrm{K}), \quad C_{e}=970 \mathrm{~J} /(\mathrm{kg} \mathrm{K}), \quad$ and $\quad \alpha_{e}=0.28$ $\times 10^{-6} \mathrm{~m}^{2} / \mathrm{s}$.

Figures 7(a) and 7(b) show the results for a constant strain rate deformation of $0.5 \times 10^{6} \mathrm{~s}^{-1}$ under an imposed pressure of $0.3 \mathrm{GPa}$. These plots demonstrate the effect of accounting for the stiffening and/or increased dissipation of the binder under the imposed pressure. Each plot shows the shear stress and temperature history at the interface between the explosive and the binder during the course of the deformation.

When pressure-augmented dissipation is not accounted for [Fig. 7(a)], the temperature near the interface increases uniformly with time but does not rise to values sufficient for 

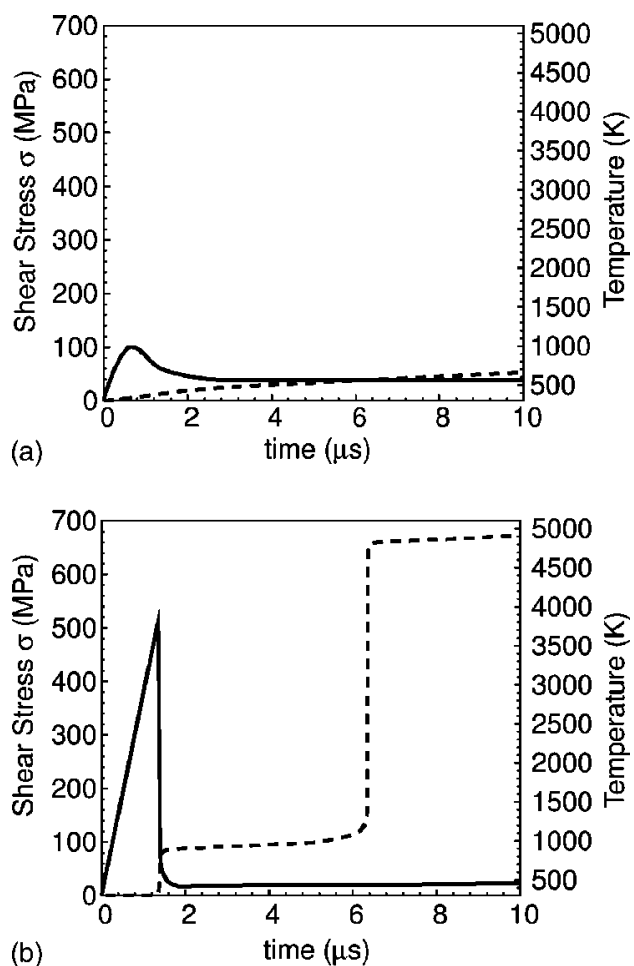

FIG. 7. Shear stress (solid curve) and temperature (broken curve) histories at the binder/explosive grain interface with $P=0.3 \mathrm{GPa}$ and $\dot{\epsilon}_{0}=0.5$ $\times 10^{6} \mathrm{~s}^{-1}$ for the small deformation analysis. (a) No pressure sensitivity of viscoelastic response. (b) Pressure sensitivity included. Including pressure sensitivity significantly alters the shear response and causes a rapid temperature rise in the binder upon catastrophic softening of the binder.

initiating any significant chemical decomposition of the explosive in the time of $10 \mu \mathrm{s}$. On the other hand, when the pressure-induced stiffening or dissipation increase is taken into account [Fig. 7(b)], a much higher shear stress is initially sustained by the binder because of the pressureinduced stiffening but initially with only very little dissipation and a correspondingly small temperature rise. However, around $1.5 \mu \mathrm{s}$, the binder softens rapidly under the influence of the rising temperature, leading to a fast and significant further heating that arises from the inelastic dissipation of most of the energy stored "elastically" in the binder until then. This temperature increase to about $900 \mathrm{~K}$ is sufficient to set off the chemical energetics of the explosive so as to lead to a "thermal explosion" at around $6 \mu \mathrm{s}$. As the energetic material is exhausted by decomposition, the reaction progresses to completion, and the thermal explosion subsides.

It should be noted here that this drop in the shear carrying capacity of the binder at $\approx 1.5 \mu \mathrm{s}$ is possibly relevant to the experimental observations reported by Field and co-workers ${ }^{10,12,13}$ on the catastrophic load drop for PBX in connection with drop-weight tests. As discussed in the introduction, these researchers concluded that this "catastrophic" failure of polymers along localized bands is responsible for their "sensitizing action" in explosives, without further specifying the nature of this "sensitization."

Figures 8(a), 8(b), 9(a), and 9(b) represent excerpts of a parameter study with respect to the relevant loading parameters, pressure, and shear deformation rate. Having estab-
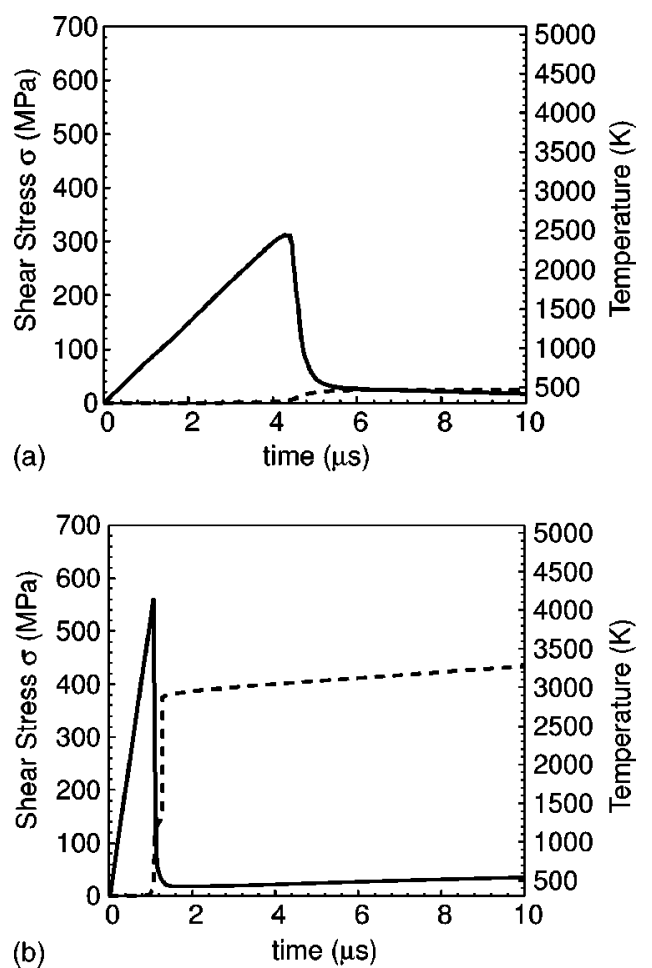

FIG. 8. Shear stress (solid curve) and temperature (broken curve) histories at the binder/explosive grain interface for the small deformation analysis with pressure sensitivity and with $P=0.3 \mathrm{GPa}$. Effect of strain rate. (a) $\dot{\epsilon}_{0}$ $=0.1 \times 10^{6} \mathrm{~s}^{-1}$. (b) $\dot{\epsilon}_{0}=0.7 \times 10^{6} \mathrm{~s}^{-1}$.
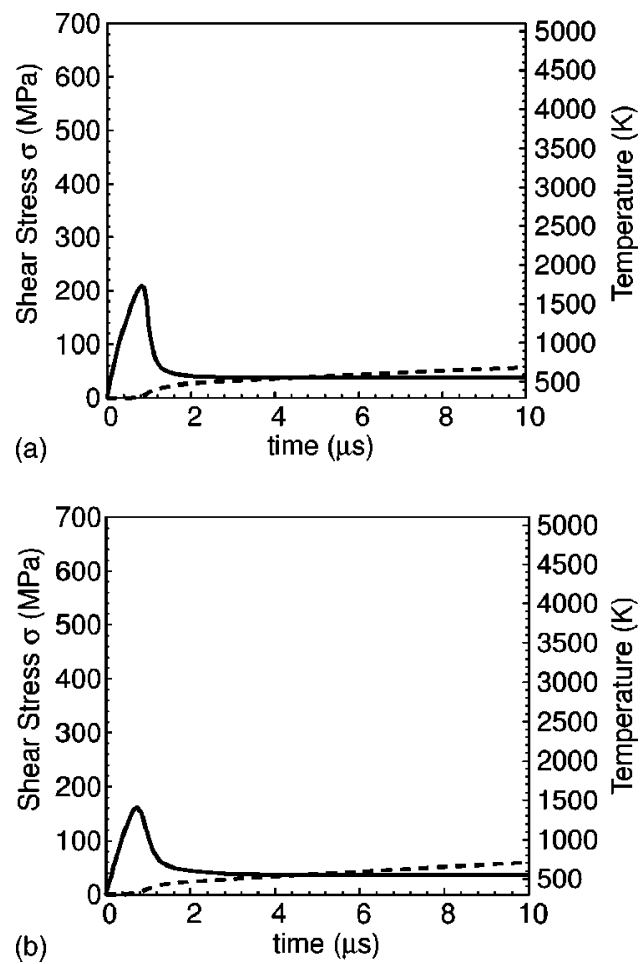

FIG. 9. Shear stress (solid curve) and temperature (broken curve) histories at the binder/explosive grain interface for the small deformation analysis with pressure sensitivity and with $\dot{\epsilon}_{0}=0.5 \times 10^{6} \mathrm{~s}^{-1}$. Effect of pressure (a) $P=0.2 \mathrm{GPa}$. (b) $P=0.1 \mathrm{GPa}$. 
lished the necessity to properly account for pressureaugmented dissipation in the rubbery binder, these computations all include pressure sensitivity. Figures 8(a) and 8(b) show, respectively, the effect of a lower and higher shear deformation rate relative to that represented in Fig. 7(b) with the pressure held at $0.3 \mathrm{GPa}$. With the lower deformation rate [Fig. 8(a)] insufficient elastic energy has accumulated when the shear stress drops to cause the temperature to rise as high as in Fig. 7(b). The temperature rise is significant $\left(\approx 125{ }^{\circ} \mathrm{C}\right)$ but is insufficient to activate the chemical energetics. At a higher deformation rate [Fig. 8(b)], more elastic energy has accumulated at the time the binder softens rapidly and hence the temperature rise is higher and the energetics are sped up as well. Figures 9(a) and 9(b) show the effect of pressure with the same shear deformation rate as Fig. 7(b). As the pressure reduces, the stiffening and dissipation effects reduce considerably, also. Higher pressures are not considered up to this point in the discussion because the constitutive formulation needs modification to account for other inelastic deformation mechanisms at the higher stress levels caused by the greater stiffening at higher pressures. This point is discussed subsequently in more detail.

\section{FINITE DEFORMATION ANALYSIS OF BINDER DEFORMATION}

The small deformation analysis presented in the previous sections allows an understanding of the main characteristics of the shear response of the viscoelastic binder under combined pressure and shear loading. In view of the large deformations experienced by the binder under these loading conditions, it is appropriate to also inquire whether finite deformation effects change the results significantly. However, today there is no generally accepted or experimentally verified constitutive description for large deformation viscoelastic behavior, so that the following developments should be viewed as tentative in precision though basically correct in terms of global behavior. The following analysis closely follows that of Tong et al. ${ }^{35}$ to which publication the reader is referred for more detail.

\section{A. Deformation kinematics}

We consider again the case of homogeneous shear deformation of a thin binder layer between two hard explosive grains. Let $\lambda(t)$ and $\kappa(t)$ represent the stretch and shear of the binder at any time during the deformation (Fig. 10) so that the deformation gradient tensor can be written as

$$
\mathbf{F}=\left(\begin{array}{ccc}
\lambda(t) & 0 & 0 \\
-\kappa(t) & 1 & 0 \\
0 & 0 & 1
\end{array}\right)
$$

From the deformation gradient tensor the spatial velocity gradient is obtained as

$$
\mathbf{L}=\dot{\mathbf{F}} \mathbf{F}^{-1}=\mathbf{D}+\mathbf{W},
$$

where $\mathbf{D}=\left(\mathbf{L}+\mathbf{L}^{T}\right) / 2$ is the rate of deformation tensor, and $\mathbf{W}=\left(\mathbf{L}-\mathbf{L}^{T}\right) / 2$ is the spin rate tensor.

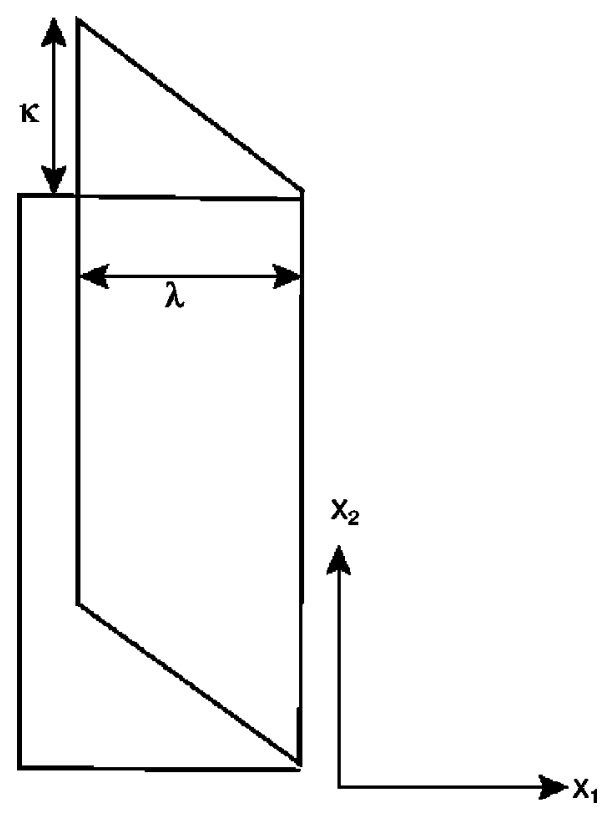

FIG. 10. Configuration for finite deformation analysis of binder shearing. $\kappa$ and $\lambda$ represent the two kinematic measures of deformation of the binder, respectively, the shear and the stretch.

The first Piola-Kirchhoff stress tensor $\mathbf{T}$ is related to the Cauchy stress tensor $\sigma$ by

$$
\sigma=\frac{1}{J} \mathbf{F} \mathbf{T}^{T},
$$

where $J=\operatorname{det} \mathbf{F}$. From the symmetry of the Cauchy stress, one obtains

$$
\mathbf{F T}^{T},=\mathbf{T F}^{T},
$$

which identifies the first Piola-Kirchhoff stress tensor to be of the form

$$
\mathbf{T}=\left(\begin{array}{ccc}
T_{11} & \kappa T_{11}+\lambda T_{21} & 0 \\
T_{21} & T_{22} & 0 \\
0 & 0 & T_{33}
\end{array}\right) .
$$

\section{B. Constitutive relations and rate equations}

We make the typical large-definition assumption that the deformation gradient tensor allows a multiplicative decomposition into elastic and inelastic (viscous) components of the form

$$
\mathbf{F}=\mathbf{F}^{e} \mathbf{F}^{v} .
$$

The above representation is refined by assuming that in a viscoelastic material there exist several 'independent' but similar and concurrently active relaxation mechanisms, each of which is governed by the overall deformation gradient $\mathbf{F}$. Thus we write ${ }^{36}$

$$
\mathbf{F}=\mathbf{F}_{i}=\mathbf{F}_{i}^{e} \mathbf{F}_{i}^{v},
$$

where $\mathbf{F}_{i}^{e}$ and $\mathbf{F}_{i}^{v}$ represent the elastic and viscous components of individual mechanisms identified by the subscript $i$. Unless indicated, there is no sum over the subscript $i$. The physical motivation for this representation follows from the 
Maxwell-Wiechert model used in the small deformation analysis (see Fig. 5).

The multiplicative decomposition of $\mathbf{F}$, together with Eq. (28) then leads to

$$
\mathbf{D}=\mathbf{D}_{i}=\mathbf{D}_{i}^{e}+\mathbf{D}_{i}^{v},
$$

which can be rewritten as

$$
\mathbf{D}_{i}^{e}=\mathbf{D}-\mathbf{D}_{i}^{v} .
$$

Thus if the viscous component $\mathbf{D}_{i}^{v}$ of the rate of deformation tensor $\mathbf{D}_{i}$ is related to the stress tensor using an appropriate inelastic flow law, and the elastic component $\mathbf{D}_{i}^{e}$ is related to the stress rates, one can obtain the evolutionary rate equations for the components of the stress tensor.

Rather than assuming a general Neo-Hookean hyperelastic response, we employ a linear response for the elastic behavior in this analysis. As discussed later, this assumption does not significantly affect the features of the behavior of the binder under the loading conditions considered; at the same time it minimizes the complexity of the formulation and focuses attention on the more relevant relaxation behavior.

The viscous flow rule for each relaxation mechanism $i$ is taken to be in the form of the associated flow law

$$
\mathbf{D}_{i}^{v}=\dot{\gamma}_{i}^{v} \frac{\mathbf{S}_{i}}{2 \sigma_{i}^{e f f}},
$$

where $\mathbf{S}_{i}=\sigma_{i}-1 / 3\left(\operatorname{trace} \sigma_{i i}\right) \mathbf{I}$ is the deviatoric stress tensor, $\sigma_{i}^{e f f}=\sqrt{1 / 2 S_{i}^{k l} S_{i}^{k l}}$ (sum over $k$ and $l$ ) is the effective stress, and $\dot{\gamma}_{i}^{v}$ is the viscous or plastic strain rate function.

The specific form of the plastic strain rate function $\dot{\gamma}_{i}^{v}$ is obtained by again referring to the Maxwell-Wiechert model from which we may write

$$
\mathbf{D}_{i}^{v}=\frac{\mathbf{S}_{i}}{G_{i} \tau_{i}(t)},
$$

which is the large deformation version of Eq. (14).

Comparing this relation to Eq. (36) above renders

$$
\dot{\gamma}_{i}^{v}=\frac{2 \sigma_{i}^{e f f}}{G_{i} \tau_{i}(t)},
$$

where, as before, $\tau_{i}(t)=\tau_{i}^{0} a_{T, P}(t)$ are the temperature and pressure-dependent relaxation times.

The resulting rate equations for the components $T_{i}^{\alpha \beta}$ of the first Piola-Kirchhoff stress tensor $\mathbf{T}_{i}$ for each mechanism $i$ can then be written as

$$
\dot{T}_{i}^{\alpha \beta}=A_{i}^{\alpha \beta} \dot{\lambda}+B_{i}^{\alpha \beta} \dot{\kappa}-R_{i}^{\alpha \beta} \dot{\gamma}_{i}^{v},
$$

where

$$
\begin{aligned}
& A_{i}^{\alpha \beta}=A_{i}^{\alpha \beta}\left(\mathbf{T}_{i}, \lambda, \kappa\right), \\
& B_{i}^{\alpha \beta}=B_{i}^{\alpha \beta}\left(\mathbf{T}_{i}, \lambda, \kappa\right), \\
& R_{i}^{\alpha \beta}=R_{i}^{\alpha \beta}\left(\mathbf{T}_{i}, \lambda, \kappa\right)
\end{aligned}
$$

are lengthy algebraic expressions listed in the appendix. It can be seen that the first two terms on the right-hand side of the rate expression Eq. (39) represent the finite-deformation elastic contributions due to the overall deformation rates $\dot{\lambda}$ and $\dot{\kappa}$, and the third term represents the stress relaxation due to the viscous flow $\dot{\gamma}_{i}^{v}$. The reader is referred to Ref. 35 for more detailed discussions on this finite deformation analysis.

The overall stress in the binder is calculated as

$$
\mathbf{T}=\sum_{i=1}^{N} \mathbf{T}_{i}
$$

where $N$ is the total number of relaxation mechanisms considered (see Table I), and the overall viscous dissipation rate is given by

$$
\dot{W}^{v}=\sum_{i=1}^{N} \dot{W}_{i}^{v}=\sum_{i=1}^{N} \mathbf{S}_{i}: \mathbf{D}_{i}^{v}=\sum_{i=1}^{N} \sigma_{i}^{e f f} \dot{\gamma}_{i}^{v} .
$$

\section{Loading and boundary conditions}

In the computational results presented below, the behavior of the binder is examined for the case of wave loading by prescribing the initial deformation rates $\dot{\lambda}_{0}$ and $\dot{\kappa}_{0}$ and stressvelocity boundary conditions which result in the following rate equations for $\lambda$ and $\kappa$ :

$$
\begin{aligned}
& \dot{\lambda}=\dot{\lambda}_{0}-\frac{2}{h_{b}\left(\rho c_{1}\right)_{e}} T_{11}, \\
& \dot{\kappa}=\dot{\kappa}_{0}-\frac{2}{h_{b}\left(\rho c_{2}\right)_{e}} T_{21},
\end{aligned}
$$

with $h_{b}$ denoting the thickness of the binder layer and $\left(\rho c_{1}\right)_{e}$ and $\left(\rho c_{2}\right)_{e}$ the longitudinal and shear impedances of the explosive grains adjacent to the binder. The initial deformation rates $\dot{\lambda}_{0}$ and $\dot{\kappa}_{0}$ are related to the particle velocity of the incoming wave in the explosive grains and the thickness of the binder layer. In the interest of brevity, the derivation of these equations is not discussed but the reader is referred to the discussions of wave loading in Ref. 35 for further understanding. Equations (43) along with Eq. (39) provide a complete set of rate expressions for updating the stress components. The thermal and energetics analysis is essentially the same as that used in the small deformation analysis.

\section{Computational results and discussion}

The rate equations for stress, deformation, and temperature are again solved numerically using the finite difference technique. A time step of $1 \mathrm{~ns}$ is again used throughout and computations extend over $10 \mu s$ of deformation. The initial values for the deformation rates are taken to be $\dot{\kappa}_{0}=1.0$ $\times 10^{6} \mathrm{~s}^{-1}$ and $\dot{\lambda_{0}}=-0.3 \times 10^{6} \mathrm{~s}^{-1}$. Other values are the same as those used in the small deformation analysis.

Figures 11(a) and 11(b) show the results of the finite deformation computations using these parameters. Each plot shows the shear stress $T_{21}$ (solid curve) and temperature history at the interface between the explosive and the binder (broken curve) during the course of the deformation. The behavior is very similar to that observed for the small deformation analysis [Figs. 7(a) and 7(b)] and again demonstrate the dominating effect of the stiffening of and dissipation in 

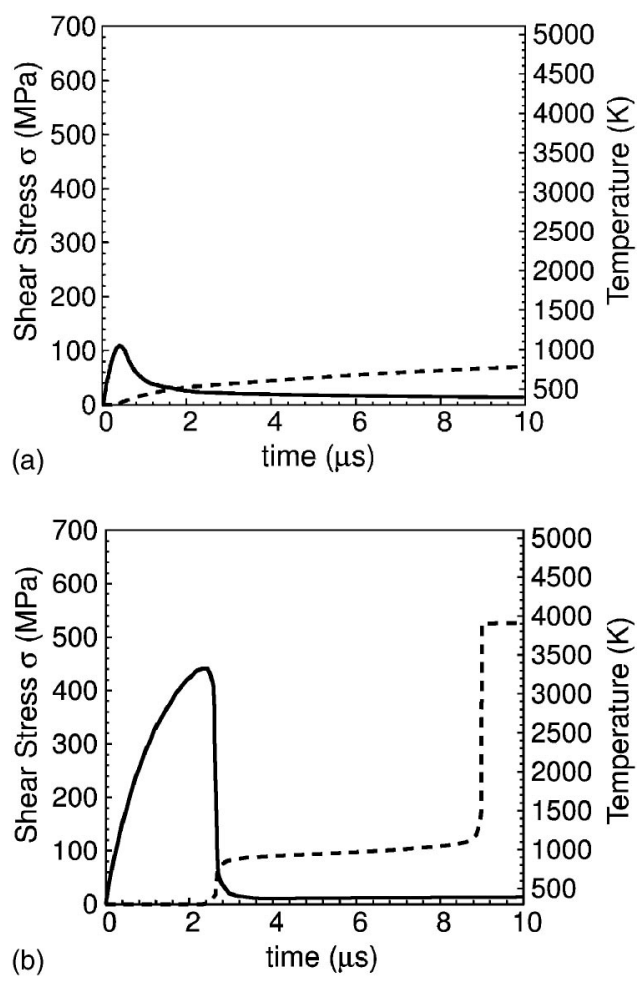

FIG. 11. Shear stress (solid curve) and temperature (broken curve) histories at the binder/explosive grain interface with $\dot{\kappa}_{0}=1.0 \times 10^{6} \mathrm{~s}^{-1}$ and $\dot{\lambda_{0}}=-0.3$ $\times 10^{6} \mathrm{~s}^{-1}$ for the finite deformation analysis. (a) No pressure sensitivity of viscoelastic response. (b) Pressure sensitivity included. The response is similar to that observed with the small deformation analysis (Fig. 7).

the binder under the (evolving) pressure. For completeness of presentation we show in Fig. 12 the evolution history of the pressure $P$, shift function $a_{T, P}$, and the shear deformation rate $\dot{\kappa}$. The pressure initially rises rapidly due to the compressive wave and induces a corresponding stiffening of the shear modulus (experienced as an increase in the value of the shift function $\left.a_{T, P}\right)$. However, in the brief time it takes the pressure to build up (by wave reverberation within the binder layer), the binder undergoes intrinsic time-dependent softening, and the accompanying viscous heating triggers a reversal in the pressure-induced stiffening of the binder (decreas-

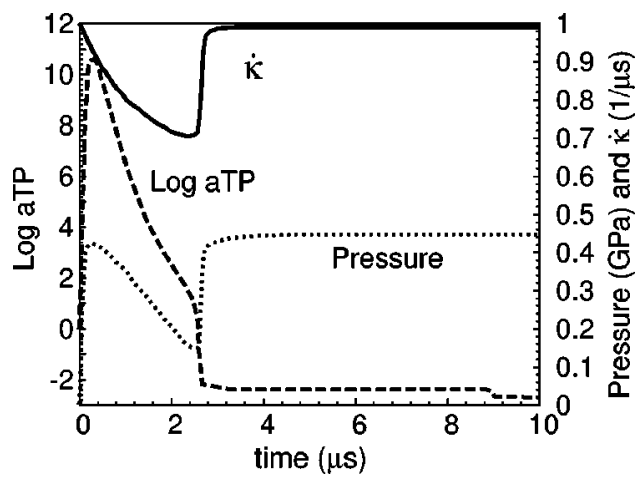

FIG. 12. Evolution of $P, \ln a_{T, P}$, and $\dot{\kappa}$ for the finite deformation analysis corresponding to the results shown in Fig. 11(b). The pressure $P$ saturates at $\approx 0.45 \mathrm{GPa}$ and the rate of shear deformation $\dot{\kappa}$ decreases from the initial loading value as the binder offers resistance and subsequently increases again as the iner softens. The shift function $a_{T, P}$ shows the initial (stiffening) due to the pressure followed by the decrease (softening) as a result of the increasing temperature. ing value of $a_{T, P}$ ). As the temperature builds up, this thermal softening is accelerated until at about $2.5 \mu$ s the binder undergoes catastrophic softening and a corresponding rapid reduction in the shear stress.

\section{FURTHER DISCUSSION}

The main objective of this work was to showcase the need for a proper accounting of the pressure sensitivity of the constitutive response of polymers apart from the relatively common considerations addressing the temperature and the intrinsically time-dependent response of binder materials. The need for a properly combined consideration of the time, temperature, and pressure-dependent response is particularly relevant to rubbery materials under shock loading conditions. During the short time-scales relevant to shock loading conditions, the normally "rubbery" material has, initially, access only to its (orders of magnitude stiffer) glassy response. However, as the temperature increases under the accompanying inelastic deformation, the material is able to access, progressively, the entire spectrum of its time-dependent response due to the strong, temperature-driven acceleration of the relaxation processes. Including the corresponding pressure-driven stiffening of the material, along with its pressure-governed viscosity, produces then a competition between the time, temperature, and pressure-dependent effects so as to generate responses that are significantly different from those predicted without such detailed considerations. In this work we have demonstrated these various competing effects by analyzing the response of the thin, rubbery binder in plastic explosives under loading conditions that occur during the initiation and propagation of detonation.

At this point it is relevant to summarize the major features of the analysis, under review of the assumptions and broad conclusions and to point to caveats derived from the presently restricted understanding of polymer behavior.

(1) Although temperature, and pressure-related nonlinearity is accounted for through a modification in the time scale, the reference master curve for the relaxation modulus is based on small-stress, small-strain, linearly viscoelastic behavior. Nonlinearity arising from larger stresses and strains is yet poorly characterized or understood in general. However, the major relevant feature is the decrease of the shear relaxation modulus by three orders of magnitude over several decades in time and hence modeling the relatively smaller nonlinear effects is not expected to change the prominent effects of the response in the analysis.

(2) To focus attention on the relaxation effects and avoid unnecessary complexity, the rubber elasticity has been modeled as linear rather than derived through a hyperelastic potential. This is justifiable because in the early stages of the deformation when the main effects (rapid fall in shear stress and corresponding viscous heating of the binder) are manifest, the short-time relaxation moduli are closer to the glassy values and hence orders of magnitude larger than any nonlinear modulus effects; at longer times when these effects may be significant, the stress levels are not high enough to render the nonlinear modulus effects significant in the overall 
context of the model. These nonlinear modulus effects are potentially less significant than the unknown nonlinear effects discussed in (1) above.

(3) Again, in an effort to keep attention focused on relaxation and dissipation effects and to minimize analytical complexity, a simplified treatment of heat conduction has been adopted. This approach is permissible, firstly because the conduction distances into the explosive grains during the time scale of the detonation process are much smaller than the binder thickness, and secondly because the sudden temperature increase due to the corresponding rapid drop in shear stress occurs over a very short period of time and hence can be considered to be (very nearly) adiabatic. The only possibly significant effect of the simplified analysis will be in the time to "thermal explosion" after this sudden temperature increase, but in this regard the analysis provides conservative (longer time) estimates for the time to explosion because peak temperatures near the binder-grain interface will be slightly higher than the average temperature used in the analysis.

\section{CONCLUSION}

The present investigations indicate that if pressureinduced stiffening or dissipation augmentation of the viscoelastic binder is accounted for along with a proper description of the time and temperature-dependent response, the mechanism of shear-induced inelastic dissipation in the explosive binder can cause the explosive to be heated locally to temperatures that are sufficient to lead to detonation within a few microseconds. This phenomenon may thus be viewed as a plausible mechanism of hot spot formation. At low shock stress levels, only a few binder layers may be oriented favorably to make this mechanism feasible and other hot spot mechanisms may be more dominant, but as detonation progresses and the shock strengthens, this mechanism may be activated at more locations. Clearly, the same mechanism cannot be invoked in the absence of pressure influence on the shear response of a rubbery binder. Further investigations need to focus on the development of typical constitutive laws of evolution and growth of hot spots in high explosives with the ultimate aim of developing experimentally supported engineering models of detonation ${ }^{37-39}$ for use in large-scale computational codes.

\section{ACKNOWLEDGMENTS}

The authors would like to acknowledge the support of the U.S. Department of Energy through the ASCI-ASAP "Center for the Dynamic Response of Materials" Contract No. B341492 under DOE Contract No. W-7407-ENG-48 at the California Institute of Technology. The authors would also like to thank Professor J. E. Shepherd at Caltech for repeated helpful discussions during this work, to D. Nelson and J. Campbell for preparing the final draft, and to $\mathrm{S}$. Browne for help in preparing print-worthy figures.

\section{APPENDIX}

Expressions for nonzero terms of $A_{i}^{\alpha \beta}, B_{i}^{\alpha \beta}$, and $R_{i}^{\alpha \beta}$ are listed below. Further details can be found in Ref. 35. For convenience and clarity, the subscript $i$ denoting individual relaxation elements is omitted in all terms.

$$
\begin{array}{rl}
A^{11}= & \frac{T_{11}}{\lambda}+\frac{2 \mu^{e}+\lambda^{e}}{\lambda^{2}}, \\
A^{22}= & \frac{\lambda^{e}}{\lambda}, \\
A^{33}= & \frac{\lambda^{e}}{\lambda}, \\
B^{21}= & \frac{T_{11}}{\lambda}+\frac{\mu^{e}}{\lambda^{2}}, \\
B^{22}= & T_{21}+\frac{\kappa}{\lambda} T_{11}+\frac{\kappa}{\lambda^{2}} \mu^{e}, \\
R^{11}= & \frac{1}{\lambda \sigma^{e f f}}\left(\lambda S_{11} T_{11}+S_{21} S_{21}+\mu^{e} S_{11}\right), \\
R^{33}= & \frac{1}{\sigma^{e f f}} S_{33}\left(T_{33}+\mu^{e}\right) . \\
R^{21}= & \frac{1}{2 \lambda \sigma^{e f f}}\left(4 P S_{21} T_{11}-2 T_{33} S_{21}+2 \mu^{e} S_{21}\right), \\
& \left.\left.+\kappa S_{21}\right)\right], \\
R^{e f f} & 1 \lambda S_{22} T_{22}+\kappa \lambda S_{21} T_{11}+\lambda S_{21} S_{21}+\mu^{e}\left(\lambda S_{22}\right.
\end{array}
$$

${ }^{1}$ See, for example, C. H. Johansson and P. A. Persson, Detonics of High Explosives (Academic, New York, 1970).

${ }^{2}$ By way of notation, the term "explosive" will be taken to refer to the composite explosive and the constituent phases will be referred to as "explosive grain" and "binder."

${ }^{3}$ J. E. Field, N. K. Bourne, S. J. P. Palmer, and S. M. Walley, Philos. Trans. R. Soc. London, Ser. A 339, 269 (1992).

${ }^{4}$ J. E. Field, G. M. Swallowe, and S. N. Heavens, Philos. Trans. R. Soc. London, Ser. A 382, 231 (1982).

${ }^{5}$ R. E. Winter and J. E. Field, Proc. R. Soc. London, Ser. A 343, 399 (1975).

${ }^{6}$ R. B. Frey, proceedings of the Seventh Symposium (International) on Detonation, 1981.

${ }^{7}$ J. K. Dienes, Mater. Res. Soc. Symp. Proc. 24, 373 (1984).

${ }^{8}$ J. G. Bennett, K. S. Haberman, J. N. Johnson, B. W. Asay, and B. F. Henson, J. Mech. Phys. Solids 46, 2303 (1998).

${ }^{9}$ V. Boyle, R. Frey, and O. Blake, proceedings of the Ninth Symposium (International) on Detonation, 1989.

${ }^{10}$ G. M. Swallowe and J. E. Field, Proc. R. Soc. London, Ser. A 379, 389 (1982).

${ }^{11}$ M. E. Kipp, J. W. Nunziato, R. E. Setchell, and E. K. Walsh, proceedings of the Seventh Symposium (International) on Detonation, 1981.

${ }^{12}$ S. N. Heavens and J. E. Field, Proc. R. Soc. London, Ser. A 338, 77 (1974).

${ }^{13}$ G. M. Swallowe and J. E. Field, proceedings of the Seventh Symposium (International) on Detonation, 1981.

${ }^{14} \mathrm{~S}$. Y. Ho, proceedings of the Tenth Symposium (International) on Detonation, 1993. 
${ }^{15}$ Dr. C. Liu (Private communications). We appreciate open discussions with Dr. Liu on this matter (Los Alamos National Laboratory, NM, USA).

${ }^{16}$ J. D. Ferry and R. A. Stratton, Kolloid-Z. 171, 107 (1960).

${ }^{17}$ M. S. Patterson, J. Appl. Phys. 35, 176 (1961).

${ }^{18}$ J. M. O'Reilly, in Modern Aspects of the Vitreous State, edited by J. D. Mackenzie (Butterworths, London, 1964).

${ }^{19}$ E. J. Perry and D. Tabor, J. Mater. Sci. 8, 1510 (1973).

${ }^{20}$ W. G. Knauss and I. Emri, Polym. Eng. Sci. 27, 87 (1987).

${ }^{21}$ G. U. Losi and W. G. Knauss, Polym. Eng. Sci. 32, 542 (1992).

${ }^{22}$ A. V. Tobolsky and H. Eyring, J. Chem. Phys. 11, 125 (1942).

${ }^{23}$ M. L. Williams, R. F. Landel, and J. D. Ferry, J. Am. Chem. Soc. 77, 3701 (1955).

${ }^{24}$ R. W. Fillers and N. W. Tschoegl, Trans. Soc. Rheol. 21, 51 (1977).

${ }^{25}$ W. K. Moonan and N. W. Tschoegl, J. Polym. Sci. A 23, 623 (1985).

${ }^{26}$ Measured at the same deformation rate or time scale.

${ }^{27}$ Y. M. Gupta, J. Appl. Phys. 51, 5352 (1980).

${ }^{28}$ H. Lu and W. G. Knauss, Mech. Time-Depend. Mater. 2, 307 (1999).
${ }^{29}$ W. G. Knauss and W. Zhu, Mech. Time-Depend. Mater. 6, 231 (2002).

${ }^{30}$ D. Qvale and K. Ravi-Chandar, Mech. Time-Depend. Mater. 6, 301 (2002); 8, 193 (2004).

${ }^{31}$ J. E. McKinney and H. V. Belcher, J. Research Natl. Bureau Standards, A. Phys. and Chem. 67A, 43 (1963).

${ }^{32}$ T. H. Deng and W. G. Knauss, Mech. Time-Depend. Mater. 1, 33 (1997).

${ }^{33}$ S. Sane and W. G. Knauss, Mech. Time-Depend. Mater. 5, 293 (2001).

${ }^{34}$ E. Catalano, R. McGuire, E. Lee, E. Wrenn, D. Ornellas, and J. Walton, proceedings of the Ninth Symposium (International) on Detonation, 1989.

${ }^{35}$ W. Tong, R. J. Clifton, and S. Huang, J. Mech. Phys. Solids 40, 1251 (1992).

${ }^{36}$ S. Govindjee and S. Reese, J. Eng. Mater. Technol. 119, 251 (1997).

${ }^{37}$ E. L. Lee and C. M. Tarver, Phys. Fluids 23, 2362 (1980).

${ }^{38}$ J. N. Johnson, P. K. Tang, and C. A. Forest, J. Appl. Phys. 57, 4323 (1985).

${ }^{39}$ P. K. Tang, J. Appl. Phys. 63, 1041 (1988). 